\title{
An intelligent detection and therapeutic device to support sleep apnea in infants
}

\author{
Sindu Divakaran ${ }^{1 *}$, T.Sudhakar ${ }^{1}$,Sindhiya $\mathbf{R}^{1}$, Rimisha Gupta $^{1}$, and $J$ Premkumar ${ }^{1}$ \\ ${ }^{1}$ Department of Biomedical Engineering, Sathyabama Institute of Science and Technology,Chennai, India
}

\begin{abstract}
Among the numerous sleep-disorders breathing patterns encountered by babies, such as intermittent respiration, premature apnea, obstructive sleep apnea wa sconsidered a major cause of concern. Upper airway structure, pulmonary system mechanics, etc. are only a few reasons why the babies are vulnerable to obstructive sleep disorder. An imbalance in the viscoelastic properties of the pharynx, dilators and pressure can lead to airway collapse. Low level of oxygen in blood or hypoxemia is considered a characteristic in infants with severe Obstructive Sleep Apnea (OSA). Invasive treatments like nasopharyngeal tubes, continuous positive airway pressure (CPAP), or tracheostomy are found to be helpful in most cases where infants experience sleep apnea. This paper suggests an appropriate method for long-term monitoring of obstructive sleep apnea in infants and, if any abnormalities are observed, the tool provides continuous airway pressure treatment until the abnormality is stabilized. Resilient propagation algorithm is utilised to train the datasets and produce a relevant output.
\end{abstract}

\section{Introduction:}

Pediatric sleep apnea is a sleeping condition where the infants breathing gets blocked partially or completely due to some blockage or constriction of the respiratory airway while sleeping [1]. Obstructive apnea and Central apnea which block the back of nose or throat and improper function of brain controlling breathing respectively are the common types of sleep apnea significantly seen in infants [2,3]. Mixed apneas are commonly seen in premature infants, where central apnea is followed by obstructive apnea. These blocks usually occur due to over relaxation of the throat muscles[4, 5]. It is hugely caused due to medical conditions during birth, genetic history or any problems associated with mouth and nose[5, 6].

There is always some instability in an infant's breathing which is a regular part of an infant's development [7]. There are instances of healthy infants also experiencing brief central apnea. But these are isolated events occurring during infancy $[8,9]$. Movements during sleep or sighs can also cause pause in breath with short duration $[10,11]$.

Physiologically, babies have more chances of getting sleep apnea than older children. The reported prevalence of infant OSA is difficult to estimate because of differing definitions; but published literature indicates that obstructive incidents in infancy have been characterized by a higher prevalence of PSG than older paediatric age groups. Three diagnosis procedure of questionnaire, Physical Examination and polysomnography were executed for enrolled children between 2 to 15 years. The proportion of low educational level was higher in parents who breast fed their babies than those who didn't. Multivariate analysis showed that snoring for $\geq 3$ months, male gender, obesity, breastfeeding, tonsillarhypertrophy, and adenoid hypertrophy were associated with OSAS .

Corresponding Author-*sindudiva@gmail.com
Relation between maternal OSA and fetal growth was analyzed with help of 155 pregnant women of non obese. An accelerated fetal growth is noticed when the fetus experiences an OSA during its third term of growth from this study.

Common symptoms seen during sleep are breathing through mouth, very noisy breathing, cessation in breathing, coughing or choking in sleep, uneasy and disturbed sleep, and sweating $[12,13]$. OSA has been linked to behavioral changes and sometimes unexpected infant death [14].

The usually preferred techniques to overcome this condition are anti-gastroesophageal reflux disease treatment,supraglottoplasty, adenoidectomy ,supplemental oxygen ,CPAP/BiPAP and other surgical and non- surgical methods $[15,16]$.

Our ultimate focus is to reduce the mortality rate of infants dying due to sleep apnea. This is done by carefully monitoring the infant throughout detect sleep apnea. Along with the breathing rate, other important bio signals are also simultaneously measured. This helps to keep a check on the infant's entire health condition [17, 18]. When sleep apnea is detected in an individual, continuous airway pressure therapy is automatically applied to the infant. This saves a lot of time and prevents the infant from falling to the risk of dying [18, 19].

Lemyre et.al suggests that in preterm babies who are not attached to an endotracheal tube, the Nasal intermittent positive pressure ventilation increases the effectiveness of NCPAP. Preterm babies with breathing problems are fixed to a ventilator that aids in breathing through a tube in the windpipe. Minimally invasive methods like NCPAP and NIPPV assists babies' breathing by using tubes that extend only till the nose, thus minimizing any harm to the lungs. After ventilator extubation, NCPAP and NIPPV can be utilized, thus, reducing the re insertion of endotracheal tube. The baby can breathe more comfortably when a steady pressure is given by the NCPAP to the back of the nose.

Martin et al., used the airway occlusion technique to measure the strength of the Hering-Breuer inflation 
reflex by using low pressure CPAP before and during the treatment. They observed that CPAP did not alter the inflation reflex in five of twelve preterm infants during the first 14 days. It is mentioned that short inspiratory efforts were noticed on the other 7 infants two times when they were on CPAP and 32 instances when they were taken off it which can be compared to the shortening that occurred two times during 58 occlusions when the seven infants were checked only two hours after birth. The peak inspiratory pressure produced following an occlusion sprang up to 3.4 to $7.1 \mathrm{~cm} \mathrm{H}_{2} \mathrm{O}$ in preterm infants who showed short responses. The respiratory rate dropped from 61 to 49 breaths/minute. The values obtained put forward the point that the infants were able to adjust to heavier respiratory loads when connected to a CPAP thereby removing the HeringBreuer deflation reflex.

Subramanian et.al., predicted that time and again disease and death in infants born before 8 months of pregnancy were caused by Respiratory distress syndrome (RDS. Intermittent positive pressure ventilation (IPPV) has been utilized for these babies to breathe. Otherwise, it was suggested to dispense continuous distending pressure of the lung, either as continuous positive pressure to the airway or as continuous negative pressure. The distending pressure was achieved by using any one of the methods given. A negative pressure chamber, face mask continuous positive airway pressure , nasal CPAP and negative pressure for less serious illness and endotracheal CPAP for more severe illness. The consequence was a better improved technique for babies. It was also acknowledged that the rate of pneumothorax can be increased by CDP.

Nasal continuous positive airway pressure (NCPAP) gives a positive pressure to the airways via the nose when an endotracheal tube is not available. A continuous distending airway pressure (CDP) is given continuously during the breath cycle in normally breathing infants. NCPAP is the preferred mode of non-invasive respiratory support in very low birth weight infants, soon after birth and neonatal resuscitation or after mechanical ventilation. The pressure to be used will be provided by the equipment providers. High pressure levels may increase lung volumes and improve respiratory mechanics and gas exchange in under-developed lungs, excessive pressure may directly dam the gas exchange and respiratory mechanics. A single constant pressure level for all very low birth weight infants or a personalized approach that chooses an initial pressure with gradual pressure level increase has to be adopted. Personalized increases may be steered by measuring of gas exchange, work of breathing, radiographic lung expansion.

\section{Materials and Methods:}

\subsection{Hardware Components: \\ SPO2 sensor probe}

The sensor detects the oxygen saturation or the amount of oxygen carrying molecules in the blood. It is a non invasive procedure.

\section{Respiration Sensor}

These pressure sensors detect the breath or respiration rate of the infants.

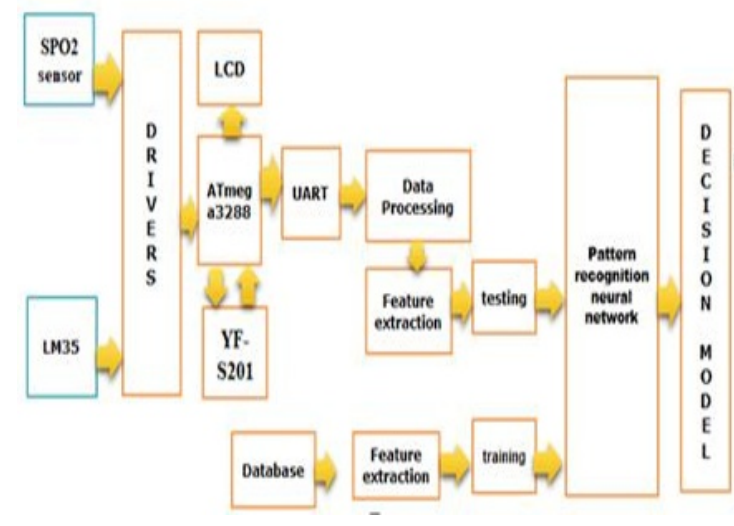

Fig 1.Block diagram of the device

\section{LM-35/Temperature Sensor}

This analog temperature sensor produces an electrical output proportional to Degree Centigrade. They provide good accuracies and need not be calibrated [23].

\section{Flow Sensor/YF-S201}

The flow sensor determines the mass flow rate of air entering through it. This is one of the most important parts of the therapy unit [24].

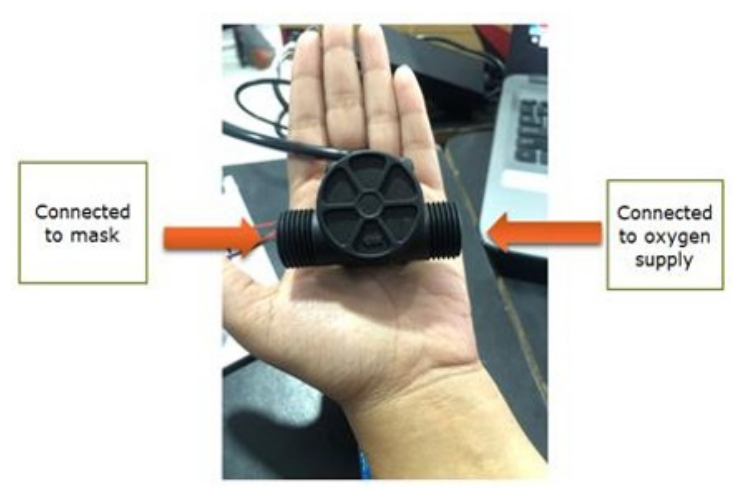

Fig 2: Flow sensor

\section{ATMEGA32}

The microcontroller employed is a 8 bit microcontroller developed on CMOS with an automatic voltage regulator based RISC architecture. 1 million instructions per second can be calculated by this microcontroller if cycle frequency is $1 \mathrm{MHz}$

\section{UART}

A receiver-transmitter is introduced in the work for asynchronous serial communication which has a configurable data format and speed of transmission. An external driver circuit takes care of the electric signaling levels [25].

LCD

A seven segment LCD display is utilized in this work to display the readings.

\subsection{Software Used:}




\section{MATLAB}

A software language for technical computing. This language does computation, programming in a platform where problems and solutions are expressed in easy to understand mathematical notations [26].

\section{Resilient propagation algorithm}

RProp is a gradient descent algorithm that makes use of thee signs of gradients to determine updates. It adapts the step size dynamically for each weight independently. This utilizes different step size for each weight.

The gradient descent variants mostly make use of the sign and the magnitude of the gradient. The gradient points are always directed to its steepest ascent. As we want to observe a minimum, we note the gradient in the opposite direction. This direction is committed to the sign of the gradient.

\subsection{Methodology:}

The various sensors are placed on the infant to measure parameters such as heart rate, $\mathrm{SPO}_{2}$, breath rate and temperature. These vital parameters are read continuously and the live data is sent in through a microcontroller through a serial port. This data is then processed and extracted. The values are then compared with the database that contains standard values. The decision model makes a decision and comes to a conclusion whether the condition of the infant is abnormal (sleep apnea) or not. If abnormal, the decisionmaking model sends a trigger to the flow sensor whose one end is connected to oxygen supply and the other end is connected to the mask on the patient's mouth. Once the trigger is given, the oxygen supply reaches the patient's mask. The therapy unit is stopped only when the condition turns normal.

\section{Results and Discussion:}

The system is a closed loop system. The live data from the patient is obtained continuously. The data is transferred to the system through a serial port. Tons of data are received every minute and hence only the data that is needed is segregated from the whole lot. The data is then tested and sent to the pattern recognition neural network. The data from the database is trained simultaneously and sent to the pattern recognition neural network. The live data and the pre-existing data are compared and the decision model takes a decision.

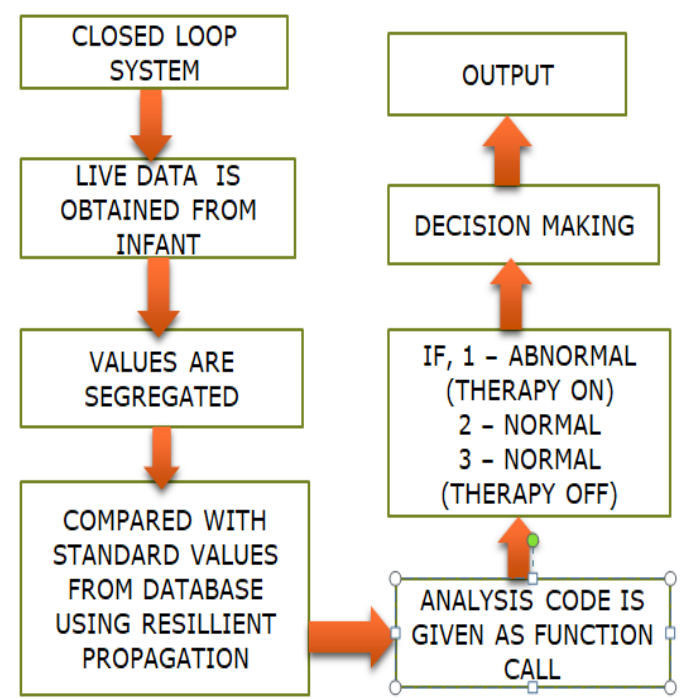

Fig 3: Flow Diagram of process

When the program is run, a pop up box appears with either numbers ' 1 ', ' 2 ' or ' 3 '. ' ' 1 ' denotes abnormality and the therapy is $\mathrm{ON}$, ' 2 ' denotes that the condition is normal and ' 3 ' denotes that the condition has come back to normal and the therapy is OFF.

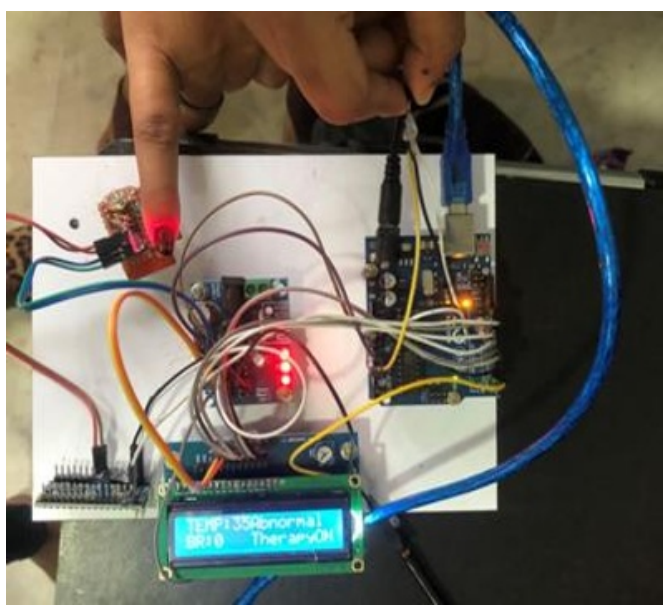

Fig 4: Work set up

Along with this, the output box has other features or plots such as performance plot, training state plot and three other plots to analyse the system. The readings of the vital parameters along with the condition whether normal or abnormal is displayed on a LCD screen. After the program is run, different plots such as performance, training state, confusion, error histogram were analysed. 


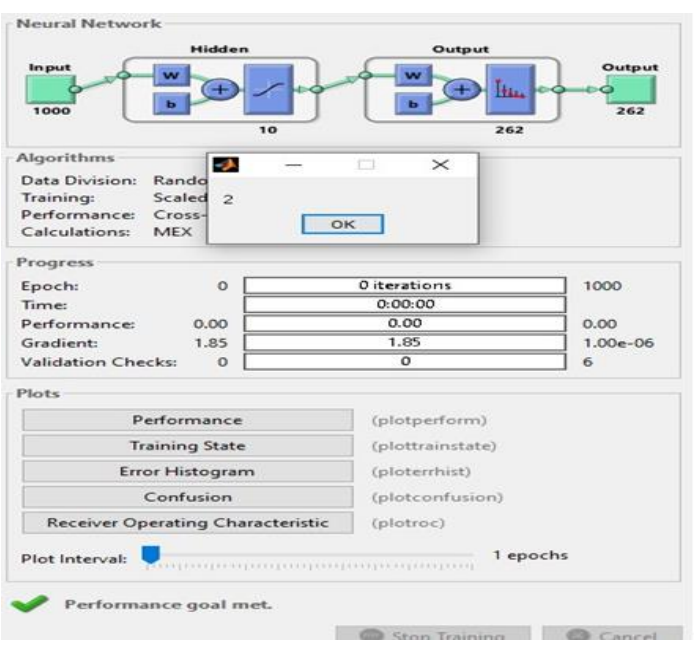

Fig 5. MATLAB output showing conditions

The output is displayed on the hardware through a liquid crystal display. The LCD keeps running a loop by displaying the readings of the vital parameters one after the other. Also, it displays the condition of the infant whether abnormal or normal.

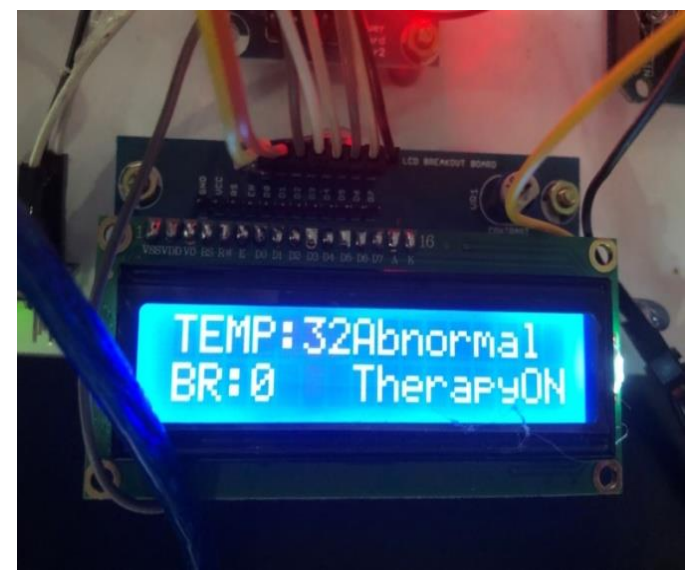

Fig 6:LCD display when the condition is abnormal

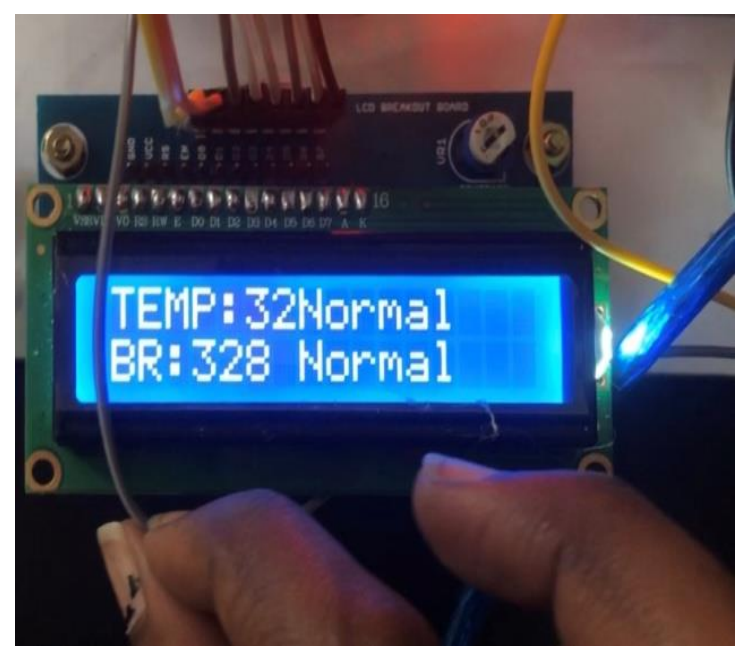

Fig 7:LCD display when the condition is normalised after therapy
Performance plot is used to obtain a plot of training record error values against the number of training epochs. In the default setup, the training stops after six consecutive increases in validation error, and the best performance is taken from the epoch with the lowest validation error.

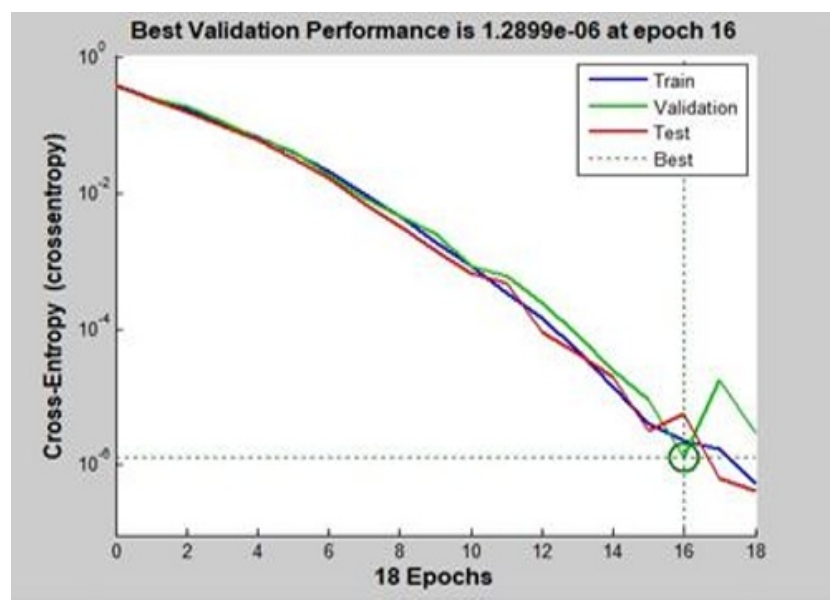

Fig 8 : Performance Plot

The current progress or status of the training at a specific time while training is in progress gives the training state. We have given 6 validation errors, hence the training will stop as soon as 6 validation errors occurs.

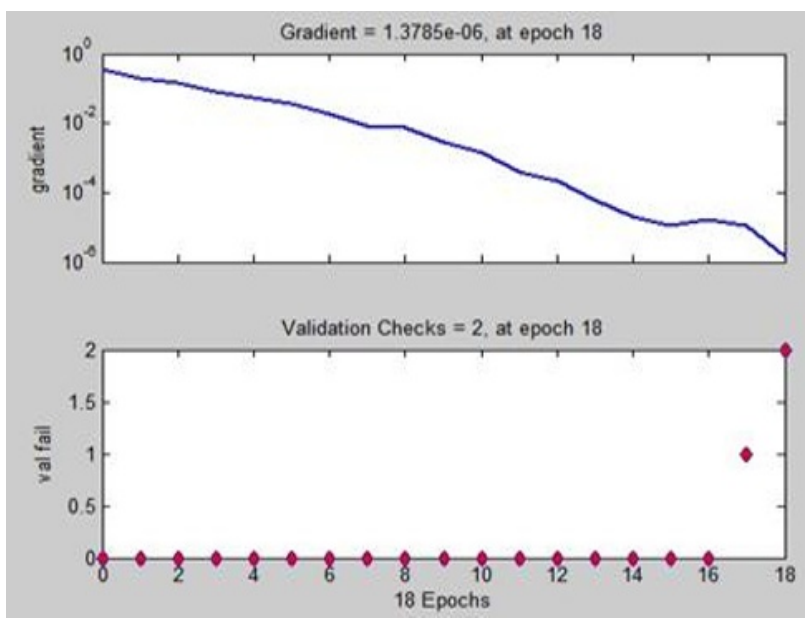

Fig 9 : Training State

The error histogram indicates the distribution of errors during testing instances in a neural network. The figure indicates the errors histogram while running our data. 


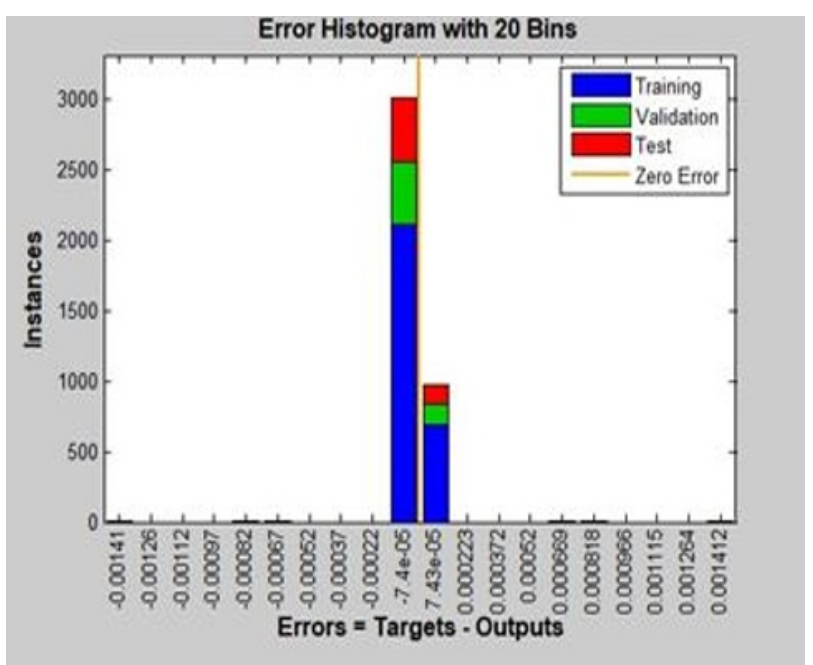

Fig 10 : Error Histogram

The total number of observations in each cell is displayed in a confusion matrix. The actual Yes and No is represented by the rows in the matrix while the column indicates the Predicted Yes or No. The correct and incorrect observations are observed in the diagonal and off-diagonal cells,respectively. Out of the data provided, it is observed that we obtained a predicted YES of 29.

Table 1 : Confusion Matrix for our data

\begin{tabular}{|l|l|l|}
\hline N=135 & $\begin{array}{l}\text { Predicted } \\
\text { YES }\end{array}$ & $\begin{array}{l}\text { Predicted } \\
\text { NO }\end{array}$ \\
\hline $\begin{array}{l}\text { Actual } \\
\text { YES }\end{array}$ & 29 & 2 \\
\hline $\begin{array}{l}\text { Actual } \\
\text { NO }\end{array}$ & 2 & 102 \\
\hline
\end{tabular}

The results obtained from running this work showed better results compared to the reviewed papers. Further it is a non- invasive technique which can be highly reliable. Inclusion of Matlab has produced desirable output. The decision model had to make the final decision on the values obtained whethe rabnormal (sleep apnea) or normal. When an abnormality related to breathing rate and oxygen level was noted by the system, it sent a trigger to the flow sensor which was connected to the oxygen supply and the mask. This trigger turned ON the therapy unit and started sending oxygen from the supply to the mask on the infant's face. The performance plot, the training state plot, confusion plot and error histogram were obtained in order to analyse the performance of the device. The therapy unit goes on till the condition of the infant becomes normal and it starts again when an abnormality is detected.

\section{Conclusions}

An effective solution has been used to detect sleep apnea in preterm infants and also therapy (NCAP) is provided when sleep apnea is detected. The results show successful analysis of the live data obtained from the infant and also shows 98.51 accuracy. The work will help in reducing mortality of preterm infants due to sleep related breathing disorders such as sleep apnea. In the future, this work will pave way for even more advanced way of detecting sleep apnea in infants and also for providing therapy.

\section{References}

1. A .Bandyopadhyay, AS Daftary. ClinPediatr (Phila). Jul;59(8):752-759(2020).

2. K. Gipson, Lu M, TB Kinane. Pediatr Rev.;40(1):313(2019).

3. W. Göpel, A. Kribs , A.Ziegler, R Laux, T Hoehn, C Wieg, et al. Lancet; 378(9803):1627-34(2011).

4. D Gozal, HL Tan, L Kheirandish-Gozal. J Clin Med. Mar 24;9(3):888(2020).

5. V Guru Anand,., G Hari Krishna,., G Mohandass,., R.J Hemalatha,.., S Sundaram,. TISC-2010, 5714621, pp. 122-124, (2010).

6. G Hari Krishnan, R.J.Hemalatha, , G. Umashankar, N Ahmed, S.R Nayak,., Adva in Int Sys and Computing 308 AISC 1, pp.635-641,( 2015).

7. G .Hari Krishnan, R.AnandaNatarajan, A. Nanda, J of E E and T, 9(6), 2114-2117, (2014).

8. R.J. Hemalatha, G.H. Krishnan, G.Umashankar, S.Abraham, Biosci Biotech Research Asia 11(2), pp.907-910,( 2014).

9. JJ .Ho, P .Subramaniam, PG.Davis Cochrane Database of Systematic Reviews 2015, Issue 7.

10. N. Ilangovan,, G. Hari Krishnan,RJPBCS, 6(3), pp.568-570, (2015).

11. G.H.Krishnan, A.Nanda, A.Natarajan, BPJ, 7(1), 221-224, (2014).

12. G.H.Krishnan, A. Natarajan,A.Nanda, BPJ, 7(1), 379-382, (2014).

13. G.H.Krishnan, R.A.Natarajan, A. Nanda, ICICES 2014, 7034178, (2015).

14. B Lemyre, PG.Davis , A.G.Paoli,H. Kirpalani . Cochrane Database of Systematic Reviews (2017), Issue 2.

15. Marta Marin-Oto, E. Eugenio Vicente, Jose M Marin, Mul Resp Med, 14(21) (2019).

16. G.Mohandass,R.AnandaNatarajan, G.Hari Krishnan, Biomedical Research (India), 26(2), pp.273-285, (2015).

17. A. Nagarjuna Reddy,G. Hari Krishnan, D. Raghuram, RJPBCS, 7(6), pp.570-575, (2016).

18. RM.Patel, S. Kandefer, M.C.Walsh, New England Journal of Medicine;372(4):331-40(2015).

19. W.F.Qubty,A. Mrelashvili, S. Kotagal . J Clin Sleep Med. Nov 15;10(11):1213-6 (2014). 
20. B Lemyre , P.G.Davis A.G.Paoli , H. Kirpalani Cochrane Database of Systematic Reviews, Issue 2.B, (2017)

21. R.J. Martin , H.S. Nearman , P.G Katona , M.H.Klaus J of Pediatrics,90(6):976-819(010).

22. J.J.Ho , P.Subramaniam , P.G.Davis. Cochrane Database of Systematic Reviews, Issue 7(2015).
23. G.M.Schmölzer ,M.Kumar, G.Pichler, K Aziz , M. O'Reilly, P.Y.Cheung, BMJ; 347:f5980 (2013).

24. A.Telerant, G.L. Dunietz, A. Many, Sci Rep 8, 10768 (2018).

25. K.I.Wheeler ,C. Klingenberg , C.J Morley,P.G Davis . Neonatology; 100(3):219-27 (2011).

26. Z. Xu , Y. Wu , J.Tai . J Otolaryngol Head Neck Surg.;49(1):11(2020). 